\title{
Lost in Translation: Psychologische Mechanismen und Psychotherapie
}

\author{
Herta Flor ${ }^{a, b}$ \\ a Institut für Neuropsychologie und Klinische Psychologie, Zentralinstitut für Seelische Gesundheit, Medizinische Fakultät Mannheim, \\ Mannheim, Deutschland; \\ bUniversität Heidelberg, Heidelberg, Deutschland
}

\section{Schlüsselwörter}

Psychotherapie - Psychologie - Verhaltenstherapie . Neurowissenschaften · Psychische Störungen

\section{Zusammenfassung}

In diesem Artikel möchte ich zu den folgenden Punkten Stellung nehmen: 1. Psychische Störungen basieren auf psychologischen Mechanismen und müssen aus diesen heraus verstanden werden 2. Psychische Störungen sind keine Hirnerkrankungen - jedoch kann das Verständnis psychischer Störungen durch die Kenntnis biologischer Prozesse verbessert werden. 3. Psychotherapie muss an diesen Mechanismen ansetzen und sollte von einer störungsorientierten zu einer mechanismenorientierten Therapie kommen. 4. Dies setzt eine mechanismenorientierte Diagnostik voraus. Der RDoc-Ansatz ist ein wichtiger Versuch, aber er greift zu kurz, was die psychologischen Mechanismen betrifft. 5. Pharmakotherapie und andere somatische Therapien können Psychotherapie unterstützen, wenn sie zur Veränderung der psychologischen Mechanismen eingesetzt werden. 6. Psychotherapie kann auch bei organischen Erkrankungen wirksam eingesetzt werden. 7. Wir sind noch weit davon entfernt, die Erkenntnisse der Grundlagenforschung in der Psychologie und den Neurowissenschaften in die Konstruktion neuer Psychotherapien einzubauen. Dafür brauchen wir bessere Förderinstrumente als die bislang zur Verfügung stehenden und eine politische und finanzielle Priorität für die Therapieforschung.

\author{
Keywords \\ Psychotherapy · Psychology · Behavior therapy · \\ Neurosciences - Mental disorders
}

\section{Summary}

Lost in Translation: Psychological Mechanisms and Psychotherapy

In this article the following points will be addressed: 1. Mental disorders are based on psychological mechanisms and have to be understood on this basis. 2. Mental disorders are not disorders of the brain; however, mental disorders can be better understood by including biological variables. 3. Psychotherapy must be based on these mechanisms and should move from a disorderspecific to a mechanism-based approach. 4. This needs a mechanism-based assessment that should go beyond the proposed research domain criteria approach. 5. Pharmacological and other somatic treatments can improve psychotherapy by enhancing psychological change. 6. Psychotherapy is also effective in many somatic disorders, and its indications need to be expanded. 7. We need to make psychotherapy research and the use of psychotherapeutic methods a political and financial priority.

\section{KARGER \\ Fax +497614520714

\section{(c) 2015 S. Karger GmbH, Freiburg}

$1016-6262 / 15 / 0252-0111 \$ 39.50 / 0$
Prof. Dr. Herta Flor 


\section{Einleitung}

Die Grundthese dieses Beitrags ist, dass die gesamte Psychologie als die Wissenschaft des Erlebens und Verhaltens die Grundlagenwissenschaft der Psychotherapie ist, wobei die klinische Psychologie in der Analyse klinischer Mechanismen eine besondere Rolle spielt. Wenn man über psychologische Mechanismen der Psychotherapie spricht, könnte man sich auf unterschiedliche Aspekte konzentrieren wie z.B. Mechanismen der Entstehung und Aufrechterhaltung psychischer Störungen, Mechanismen des therapeutischen Prozesses und der Mediation des Therapieeffektes oder Mechanismen des Erfolgs einer Therapie. In all diesen Fällen tragen psychologische Mechanismen entscheidend zum Verständnis bei; ich möchte mich hier jedoch auf das Thema der Entstehung und Aufrechterhaltung psychischer Störungen konzentrieren. Dies möchte ich anhand von Beispielen aus der Literatur aber auch aus unseren eigenen Forschung in Sonderforschungsbereich 636 der Deutschen Forschungsgemeinschaft (DFG) tun, in dem wir unter dem Thema 'Learning, memory and brain plasticiry: implications for psychopathology' einen mechanismenbasierten Ansatz zum Verständnis psychischer Störungen ausgearbeitet und untersucht haben. Schwerpunkt sind hier die Themen Angst, Abhängigkeiten und Depression. Auch zum Thema der psychologischen Schmerztherapie haben wir ähnliche Untersuchungen durchgeführt. Psychotherapie basiert auf dem Verständnis der psychologischen Determinanten der Psychopathologie, wobei interagierende biologische Prozesse zusätzliche Interventionsmöglichkeiten eröffnen können. Ich beschränke mich bei der Diskussion der Psychotherapie nicht auf psychische Störungen, weil sich gezeigt hat, dass auch bei organischen Störungen wie der Epilepsie oder dem Schlaganfall psychotherapeutische Interventionen sehr effektiv sind. Psychotherapie umfasst für mich somit alle Interventionen, die psychologische Methoden nutzen, also auch solche, die physiologische Prozesse beeinflussen (wie Neurofeedback bei Epilepsie oder GehirnComputer-Interfaces bei Gelähmten, siehe unten). Ich werde im Folgenden 7 Thesen diskutieren und mit Daten untermauern, die sich auf das Verhältnis der Psychologie und der Neurowissenschaften zum Verständnis psychischer Störungen und deren Behandlung stützen.

\section{Psychische Störungen basieren auf psychologischen Mechanismen und müssen aus diesen heraus verstanden werden}

In der Literatur liest man immer wieder, dass psychische Störungen Erkrankungen des Gehirns sind, zuletzt in den Ausführungen des Research Domain Criteria (RDoc) Project, das eine störungsübergreifende Analyse psychischer Störungen auf mehreren biologischen und psychologischen Ebenen vorschlägt [Cuthbert und Insel, 2013; Cuthbert und Kozak, 2013]. Im Gegensatz zu neurologischen Störungen gibt es bei den psychischen Störungen bislang keine klaren Hinweise, dass tatsächlich ein pathologischer Gehirnprozess die Grundlage der Störung ist [Lilienfeld, 2014]. Viel- mehr belegt die neurowissenschaftliche Forschung eindeutig, dass viele Hirnveränderungen durch Erleben und Verhalten erst bedingt werden, wie z.B. der Zusammenhang von Stresserleben und Hippokampus- oder Amgydalavolumen bzw. der Konnektivität präfrontaler Areale zeigt [Krugers und Joëls, 2014]. Auch die Befunde zur Bedeutung epigenetischer Faktoren im Vergleich zu genetischen Faktoren deuten in diese Richtung [Meaney und Szyf, 2006]. In einer Studie zur Initiierung von schädlichem Trinkverhalten bei Jugendlichen konnte die Varianz beispielsweise durch Gehirnaktivierungen bei einer Aufgabe, bei der unterschiedlich große Belohnungen antizipiert wurden, am wenigsten erklärt werden, während Persönlichkeitsvariablen und Verhaltensdaten, die mit der Belohnungsverarbeitung in Bezug standen, die Trinkmenge deutlich besser vorhersagten [Nees et al., 2012] (Abb. 1). Diese Befunde legen nahe, dass sich präventive Interventionen auf die Veränderung dieser Verhaltensweisen konzentrieren sollten [Conrod et al., 2013] und weniger auf somatische Aspekte.

Ein klarer Zusammenhang zwischen Grundlagenforschung und Psychotherapie zeigt sich insbesondere bei den Angststörungen, da hier die Grundlagenforschung zu Konditionierungsprozessen, Extinktionslernen oder zu kognitiven Verzerrungen im großem Umfang Eingang in die therapeutische Praxis gefunden hat [Craske et al., 2014]. Bei anderen therapeutischen Ansätzen, wie solchen aus der «3. Welle» der kognitiven Verhaltenstherapie, lassen sich die Zielvariablen und wirksamen Elemente ebenso den entsprechenden psychologischen Grundlagen zuordnen [Hofmann und Asmundson, 2008]. Bei der Evaluation der Übertragung grundlegender psychologischer Erkenntnisse zu Störungen in die Psychotherapie muss man bedenken, dass die klinische Psychologie eine relativ junge Wissenschaft ist und viele Interventionen erst in den letzten 50 Jahren entwickelt wurden [Barlow, 2014]. Es ist jedoch unübersehbar, dass in der Umsetzung psychologischer Erkenntnisse in die Psychotherapie eine große Lücke klafft, die nicht nur wissenschaftlicher sondern auch politischer Anstrengungen bedarf (siehe dazu Punkt 7 unten).

\section{Das Verständnis psychischer Störungen kann durch die Kenntnis biologischer Prozesse verbessert werden}

Gerade die neurowissenschaftliche Forschung hat einen wichtigen Beitrag zum Verständnis psychischer Störungen geliefert, da wichtige grundlegende Konzepte der Störungsforschung in den Neurowissenschaften aus der Psychologie gekommen sind. Das kann man sehr gut in der Forschung zum abhängigen Verhalten sehen, wo Konzepte wie maladaptive Lernprozesse und damit einhergehende motivationale Veränderungen, z.B. in der Anreiz-Motivations-Theorie der Sucht [Robinson und Berridge, 1993] dargestellt, zu wichtigen neuen Erkenntnissen geführt haben. So wurde postuliert, das bei Abhängigkeit mit der Belohnungsverarbeitung assoziierte Hirnareale nicht nur durch die Abhängigkeit induzierende Substanz selbst, sondern auch über Lernprozesse assoziierte interne und externe Reize aktiviert werden können und Drogen- 


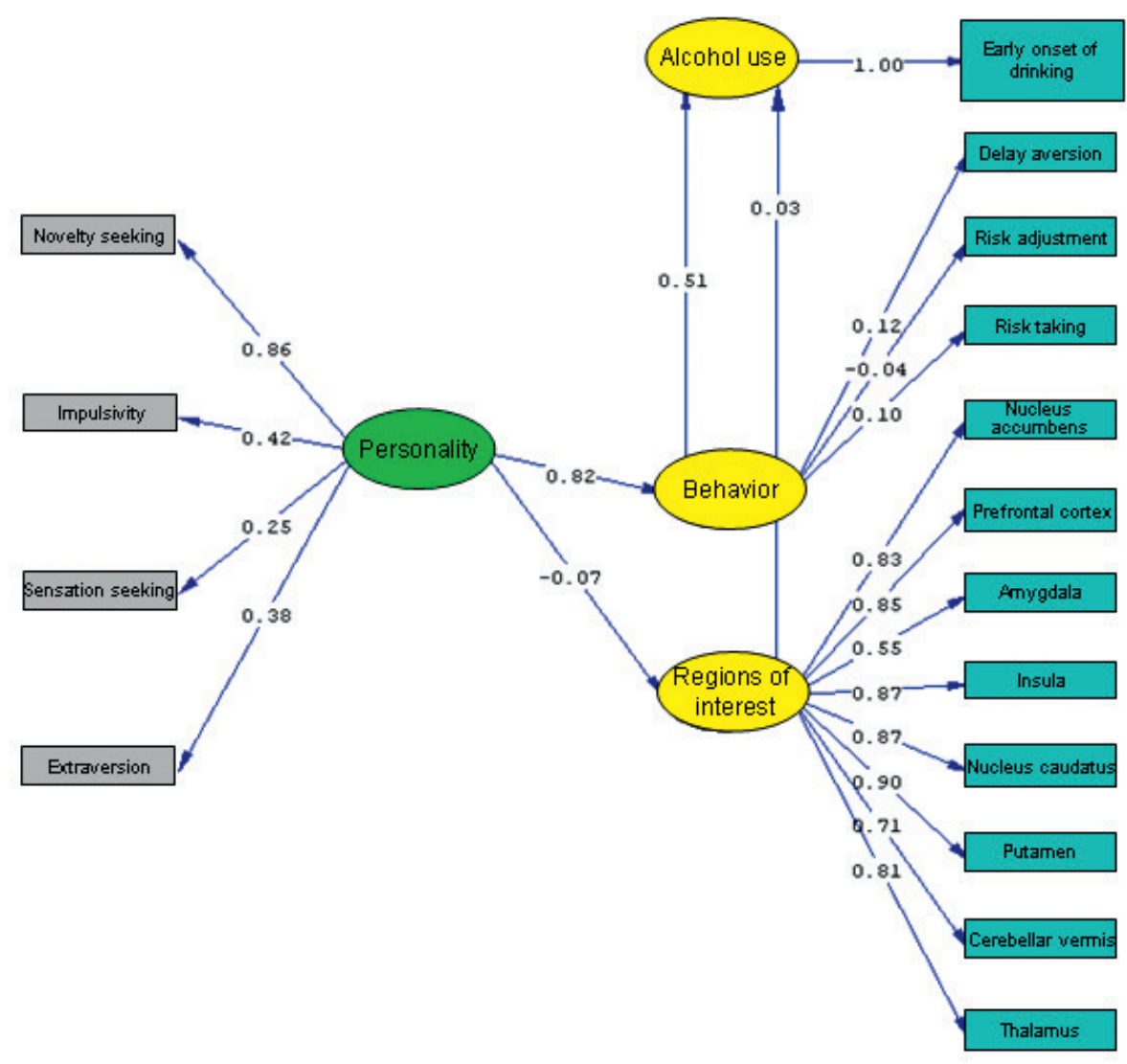

Abb. 1. Vohersage frühen Trinkverhaltens bei Jugendlichen auf der Basis von Persönlichkeitsvariablen, neuropsychologischen Tests und der Hirnaktivierung auf eine Aufgabe zur Belohnungsantizipation.

nahelegt, dass auch hier Lernprozesse entscheidend an der Aufrechterhaltung beteiligt sind [Wölfling et al., 2011]. Ferner konnten wir einen Zusammenhang zwischen der verminderten Schreckreflexreaktion bei Präsentation alkoholassoziierter Reize und der Trinkmengen nach einem Rückfall zeigen. Ähnliche Zusammenhänge zeigten sich, wenn mittels funktioneller Magnetresonanztomograhie (fMRT) direkt die Aktivierung der in die Belohnungsverarbeitung involvierten Hirnregionen (Striatum) analysiert wurde [Grüsser et al., 2004].

Ausgehend von diesen Überlegungen erscheint die Konfrontation des alkoholabhängigen Patienten mit kritischen alkoholassoziierten Reizen im Rahmen der Therapie eine vielversprechende Möglichkeit zur Reduktion von Alkoholverlangen und Rückfallgefährdung zu sein. Es sollte dadurch zu einer Löschung der konditionierten Reaktionen kommen. Im Rahmen des Reizexpositionstrainings erfolgte eine In-vivo-Konfrontation des Patienten mit seinem bevorzugten alkoholischen Getränk sowie eine Auslösung von Emotionen oder Gedanken, die zur Alkoholaufnahme führen (externe und interne konditionierte Reize). Dies wurde vergleichen mit einer standardisierten kognitiv-verhaltenstherapeutischen Behandlung, bei der die Auseinandersetzung des Patienten mit individuellen, kritischen Rückfallsituationen und die Erarbeitung alternativer Verhaltensstrategien zum Umgang mit diesen Situationen im Mittelpunkt stand. Sowohl im Hinblick auf die Rückfallhäufigkeit als auch auf die Variablen des Trinkverhaltens war die Therapieeffektivität der Reizexpositionsbehandlung der kognitiv-verhaltenstherapeutischen Behandlung überlegen, wenn es sich um be- 

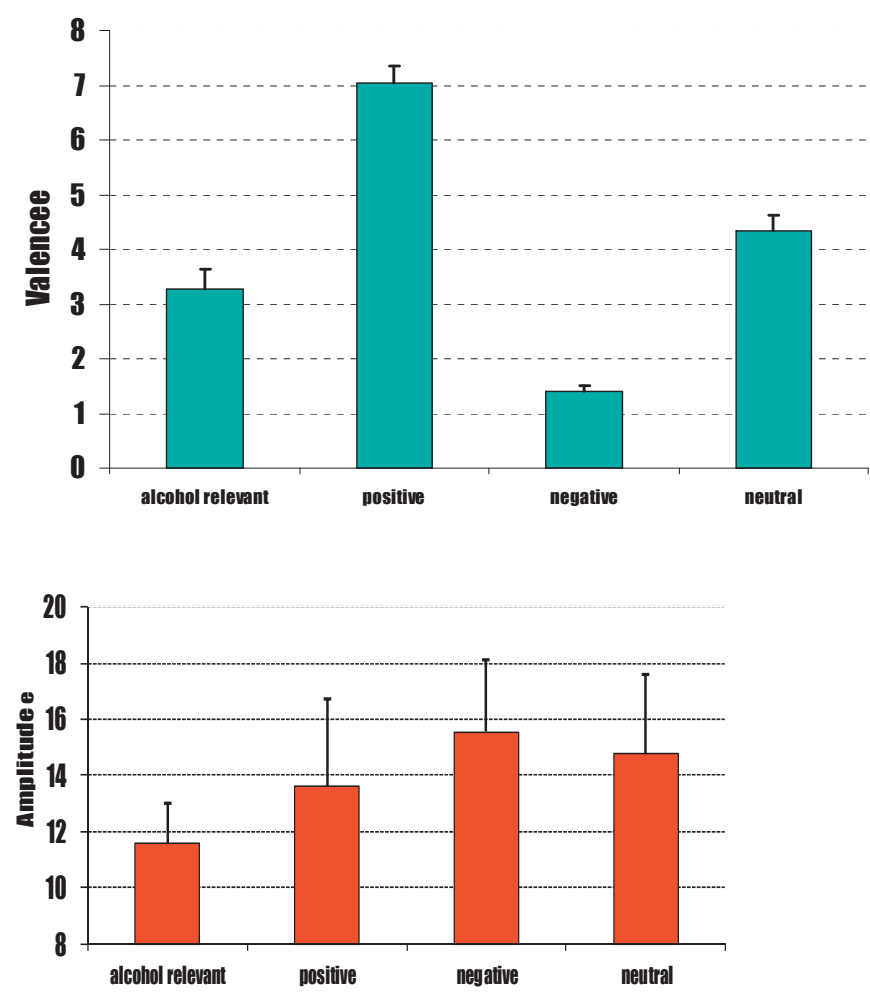

Abb. 2. Der Lidschlagreflex bei Präsentation alkoholassoziierter Reize im Vergleich zu positiven, negativen und neutralen Reizen.

sonders schwere Trinker handelte [Loeber et al, 2006] (Abb. 3). Eine weitere Fortführung dieser Untersuchungen besteht in der Anwendung einer Neurofeedbacktherapie, bei der die Hirnveränderungen bei der Exposition auf drogenrelevante Reize direkt mittels MRT zurückgemeldet werden und die Patienten somit lernen können, ihre in der Regel nicht bewussten Hirnreaktionen auf die Droge selbst zu kontrollieren [Kirsch et al., 2014]. Obwohl hier eine funktionelle organische Veränderung trainiert wird, handelt es sich um ein psychotherapeutisches Verfahren, da die Selbstregulationsfähigkeit des Patienten im Mittelpunkt steht.

\section{Psychotherapie muss mechanismenorientiert sein}

Wie oben bereits ausgeführt, muss sich eine Psychotherapie an gestörten Mechanismen orientieren und weniger an diagnostischen Kategorien, da ähnliche Mechanismen bei verschiedenen Störungen auftreten können und andererseits bei ein und derselben Störungen Untergruppen existieren können, die völlig unterschiedliche Störungsmuster aufweisen. So fanden wir z.B., dass Personen mit einer Borderline-Persönlichkeitsstörung ohne dissoziative Störungen eine eher verstärkte Konditionierung aversiver Reize aufweisen, während Personen mit Dissoziation in einem Furchtkonditionierungsparadigma nicht lernen [Ebner-Priemer et al., 2009] (Abb. 4). Ebenso finden sich bei der posttraumatischen Belastungsstörung Personen mit ausgeprägten subjektiven und physiologischen Reaktionen auf traumarelevante Reize und solche, die eher mit einer Hemmung in Verhalten und Physiologie reagieren [Wessa und Flor, 2007] und bei

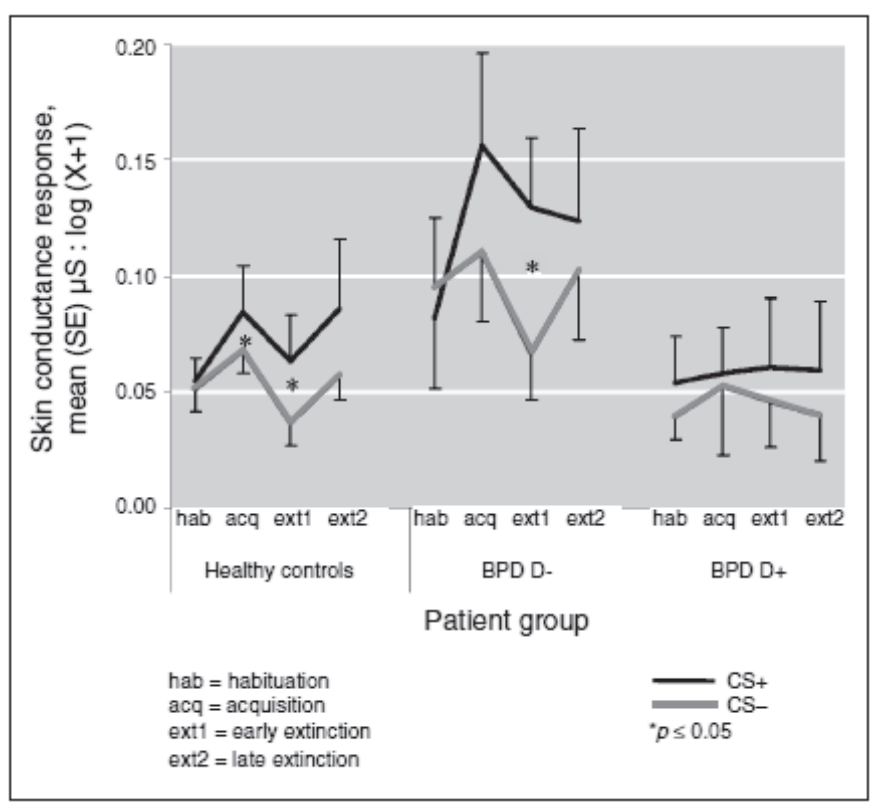

Abb. 3. Bessere Effektivität der Expositionsbehandlung bei Personen mit schwerer im Vergleich zu leichterer Alkoholabhängigkeit.

denen in unterschiedlichem Ausmaß reizbezogene Konditionierung und Kontextkonditionierung verändert sind (Steiger et al., im Druck). Hier muss bei der Therapie unterschiedlich vorgegangen werden. Transdiagnostische Therapieansätze, die spezifische gestörte Verhalten- oder Erlebensweisen angehen, sollten deshalb stärker entwickelt und untersucht werden [Rector et al., 2014]. Dies ist auch insbesondere vor dem Hintergrund der enormen Komorbidität psychischer Störungen sinnvoll, die die Heterogenität der störungsspezifisch definierten Gruppen weiter verstärkt. Dies ist auch deshalb sinnvoll, weil viele psychologische Mechanismen, die störungsrelevant sind, bei mehreren Störungen eine Rolle spielen, wie sich z.B. anhand der Furchtkonditionierung [Nees et al., im Druck] zeigen lässt. Auch die Analyse von Stressfolgen ergab ähnliche diagnoseübergreifende Befunde [Krugers und Joëls, 2014].

\section{Mechanismenorientierte Diagnostik als Voraussetzung}

Mechanismenorientierte Psychotherapie bedarf einer mechanismenorientierten Diagnostik. Die Verhaltensanalysen der Verhaltenstherapie haben diesen Ansatz schon immer gewählt, jedoch ist er in letzter Zeit zugunsten kategorialer Diagnosen in den Hintergrund getreten. Der oben bereits genannte RDoc-Ansatz ist ein wichtiger Versuch, von einer kategorialen zu einer dimensionalen Diagnostik zu kommen, jedoch greift er in mehrerer Hinsicht zu kurz. Es ist zunächst nicht zu vertreten, dass psychische Störungen als Störungen des Gehirns zu betrachten sind. Natürlich kommt es bei psychischen Störungen zu Hirnveränderungen, aber diese Veränderungen können gänzlich über psychologische Faktoren vermittelt sein, wie oben dargestellt. Andererseits kann es sicher strukturelle Veränderungen des Gehirns geben, die bestimmte 


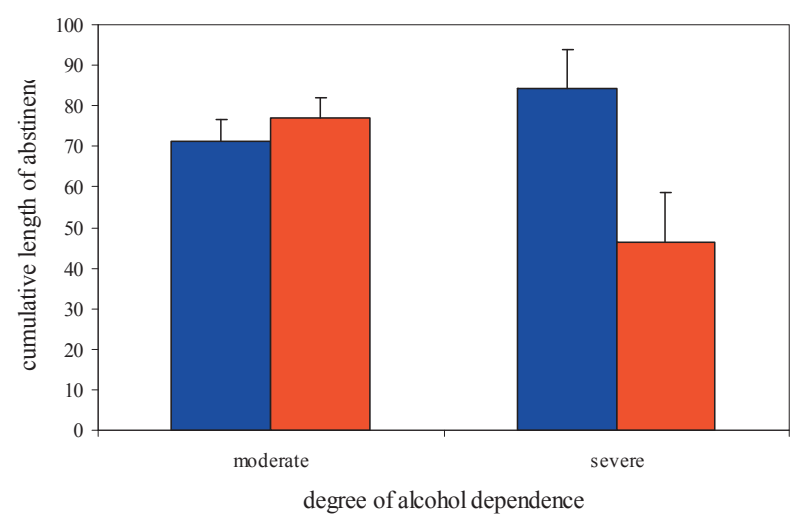

Abb. 4. Daten zur Furchtkonditionierung bei Personen mit Borderline-Persönlichkeitsstörung mit und ohne dissoziative Symptome.

Verhaltensweisen begünstigen können. So kann man bei Gesunden zeigen, dass die Fähigkeit zur Kontextkonditionierung mit dem Volumen des Hippokampus und die Fähigkeit zur Reizkonditionierung mit dem Volumen der Amygdala kovariiert [Cacciaglia et al., 2014]. Kausale Zusammenhänge lassen sich daraus jedoch nicht ableiten, da auch Stress die Volumina beider Hirnareale moduliert. Miller [2010] hat darauf hingewiesen, dass psychologische und biologische Erklärungen distinkt sind und man nicht die einen durch die anderen ersetzen kann, ohne dass man deshalb eine dualistische Perspektive einnehmen müsste. Psychologische Konstrukte wie verzögertes Extinktionslernen, Übergeneralisierung und Aufmerksamkeitsbias müssen deshalb die Grundlage einer psychologischen Diagnostik und die Grundlage für die Therapie sein.

\section{Psychotherapie kann mit somatischen Verfahren kombiniert noch effektiver sein}

Pharmakotherapie und andere somatische Verfahren wie nichtinvasive Hirnstimulation können Psychotherapie unterstützen, wenn sie spezifisch zur Verbesserung der psychologischen Veränderungen eingesetzt werden. In den letzten Jahren haben sich bei einer Reihe von kombinierten Verfahren gute Effekte nachweisen lassen. Hierzu gehören z.B. die Verwendung von D-Cycloserin zur Beschleunigung der Extinktion bei der Expositionsbehandlung von Angststörungen [Rodrigues et al., 2014], die Gabe von Glukokortikoiden bei der Behandlung der posttraumatischen Belastungsstörung [Bentz et al., 2010] oder die Verwendung von Oxytocin zur Verbesserung sozialer Kognition [Perez-Rodriguez et al., 2015]. Wichtig ist hier festzuhalten, dass diese Kombinationstherapien eine klare theoretische Grundlage haben, über einen befristeten Zeitraum Verwendung finden und sich somit auch Nebenwirkungen und andere negative Effekte von Psychopharmaka minimieren lassen. Ähnliches gilt für die Verwendung von Stimulationsverfahren wie der transkraniellen Magnetstimulation oder der D(irect) C(urrent)-Stimulation [De Raedt et al., 2014]. Wir haben auf der Basis von Befunden, die eine wichtige Bedeutung des Cannabinoid-

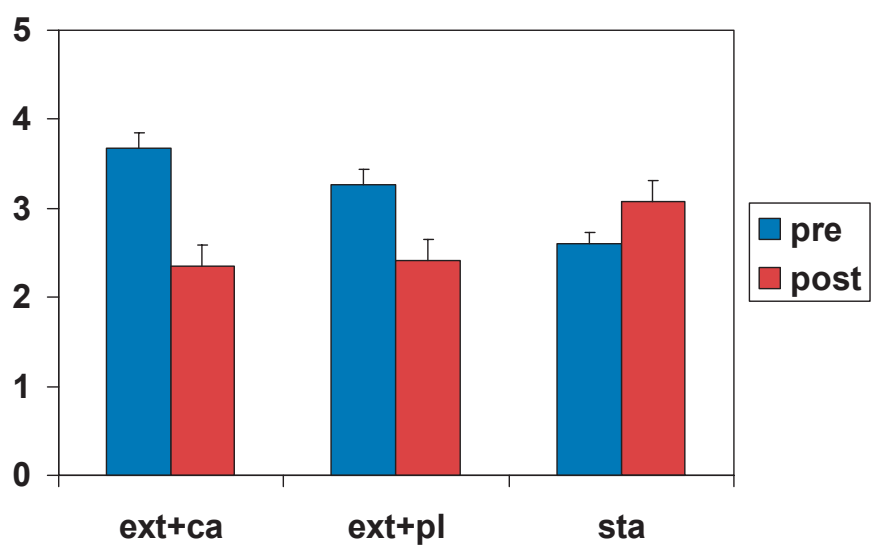

Abb. 5. Verbesserung in der schmerzbezogenen Beeinträchtigung (Multidimensionaler Schmerzfragebogen) nach Extinktionstraining (ext) mit Cannabis (ca) oder Placebo ( $\mathrm{pl})$. Die Kontrollgruppe erhielt die bestmögliche medizinische Behandlung (sta).

Rezeptors bei der Furchtextinktion nahelegen [Rabinak und Phan, 2014], Cannabis als Verstärkung eines Extinktionstrainings bei Patienten mit chronischen Schmerzen der Skelettmuskulatur eingesetzt. Es zeigte sich, dass insbesondere Patienten mit chronischen Rückenschmerzen, bei denen Lernprozesse in der Schmerzaufrechterhaltung eine wichtige Rolle spielten, von einer niedrigen Dosis Cannabis (die selbst nicht schmerzreduzierend wirkt) im Vergleich zu Placebo beim Extinktionstraining profitierten (Abb. 5).

\section{Das Potenzial der Psychotherapie ist auch bei organischen Störungen enorm und wurde bislang nicht genügend genutzt}

Bei chronischen Schmerzen hat man längst die Trennung zwischen organisch und psychisch bedingten Schmerzen zugunsten einer psychobiologischen Sichtweise aufgegeben und differenziert nicht mehr danach, ob ein organischer Befund vorliegt oder nicht; vielmehr werden behandelbare Mechanismen evaluiert. So gibt es einen breiten Konsens darüber, dass bei der Chronifizierung von Schmerz psychologische Mechanismen im Vordergrund stehen, die sich insbesondere in maladaptiven Lern- und Gedächtnisprozessen zeigen [Flor, 2014]. Angesichts dieser Befunde haben wir ein Extinktionstraining entwickelt, das auf der Basis eines operanten Ansatzes spezifische Verfahren beinhaltet, um durch die Schmerzerfahrung entstandene körperbezogene, emotionale und kognitive Veränderungen, die die Löschung der aversiver Gedächtnisinhalte behindern, zu befördern. Dazu gehören Videofeedback und Spiegeltraining, Übungen in sensorischer Diskrimination, Übungen in erweiterter Realität, Arbeit mit wichtigen Bezugspersonen und Übungen zum Aufbau schmerzinkompatibler Verhaltensweisen [Flor und Turk, 2011] (Tab. 1). Diese Verfahren können die moderaten Effekte der traditionellen Schmerzbewältigungstrainings deutlich verbessern [Thieme et al., 2007; Foell et al., 2014] und erhöhen insbesondere die Selbstkontrollfähigkeiten, verbunden mit einer abnehmenden Inanspruchnahme der Gesundheitsversorgung (Abb. 4). Dabei 
Tab. 1. Übersicht zum Extinktionstraining bei chronischen Schmerzen

Übersicht zum Extinktionstraining bei chronischen Schmerzen

Training von schmerzinkompatiblem Verhalten

Abbau von Schmerzausdruck durch Videofeedback und Spiegeltraining

Arbeit mit Bezugspersonen, um gesundes Verhalten zu belohnen

Training angenehmer Tätigkeiten

Aufbau von Arbeitsfähigkeit

Training in Medikamentenmanagement

Sensorisches Diskriminationstraining

ist es unerheblich, ob eine somatische Grundlage der Schmerzen vorliegt. Zusammen mit dem Verhalten ändert sich auch die Hirnaktivierung und normalisiert sich [Diers et al., 2012]. Psychotherapeutische Interventionen können auch bei vielen anderen als somatisch klassifizierten Störungen wie z.B. Epilepsien wirksam sein, wo Neurofeedback deutlich die Anfallsfrequenz vermindert [Birbaumer et al., 2009], und sollten auch hier eingesetzt werden. Eine besonders effektive, auf psychologischen Prinzipien basierende Intervention wurde für Patienten mit Hemiparesen nach Schlaganfällen entwickelt. Hier wurde vermutet, dass es zu einer gelernten Nichtnutzung der Extremität kommt, da im frühen Stadium des Schlaganfalls erhebliche Schwierigkeiten bestehen, Aktivitäten mit dem betroffenen Arm auszuführen. In der Therapie wird die Nutzung des Arms verhindert (constraint-induced movement therapy) und damit die Nutzung der betroffenen Hand forciert. Dies wird kombiniert mit Shaping und anderen Trainingsprozeduren. Die Effekte dieser rein psychologischen Therapie sind beachtlich [Nilsen et al., 2015; Kunkel et al., 1999; Miltner et al., 1999]. Bei Patienten ohne jegliche Mobilität der Hand wurden Gehirn-Computer-Interfaces eingesetzt, um über die Kontrolle der neuronalen Aktivität des motorischen Kortex die Beweglichkeit der Hand und des Arms wiederherzustellen [Soekadar et al., 2014]. Diese Liste ließe sich beliebig verlängern, da das Potenzial der Psychotherapie bei somatischen Störungen unendlich und bei Weitem nicht ausgeschöpft ist.

\section{Die Erforschung und Implementierung neuer Psychotherapien muss politisch unterstützt und gefördert werden}

Studien zu den Behandlungspräferenzen bei psychischen Störungen zeigen, dass $75 \%$ der Bevölkerung eine psychologische einer pharmakologischen vorziehen, wenn sie die Wahl haben, jedoch wird ihnen das oft nicht angeboten [McHugh et al. 2013]. Wir sind noch weit davon entfernt, die Erkenntnisse der Grundlagenforschung und der Neurowissenschaften in die Konstruktion neuer Psychotherapien einzubauen, und wir haben auch zu wenig Möglichkeiten, die klinische Praxis innovativer Therapien zu erproben. Dies liegt nicht nur an der medizinisch und pharmakologisch dominierten Krankenversorgung, sondern auch daran, dass zu wenig Gelder für die Erforschung von Psychotherapie im Vergleich zur Pharmakotherapie zur Verfügung stehen, weil hier eine schlagkräftige Industrie fehlt. Aber auch die öffentlichen Ausgaben spiegeln diese Imbalance wider. Obwohl psychische Störungen mehr als 15\% der Krankheitsbelastung ausmachen, werden in der EU nur 2\% des Forschungsbudgets dafür ausgegeben. Davon erhalten psychologische Verfahren lediglich 15\% [Holmes, 2014]. Die Gründung eines Instituts für Therapieforschung, das auch Mittel vergeben kann, wäre hier eine sinnvolle Maßnahme.

\section{Dank}

Diese Arbeit wurde von der Deutschen Forschungsgemeinschaft (SFB636/ C1 und Koselleck Projekt Fl 156/41-1) unterstützt.

\section{Disclosure Statement}

Die Autorin erklärt, dass keine Interessenskonflikte in Bezug auf die vorliegende Arbeit bestehen.

\section{Literatur}

Barlow DH: The neuroscience of psychological treatments. Behav Res Ther 2014;62:143-145.

Bentz D, Michael T, de Quervain DJ, Wilhelm FH: Enhancing exposure therapy for anxiety disorders with glucocorticoids: from basic mechanisms of emotional learning to clinical applications. J Anxiety Disord 2010;24:223-230.

Birbaumer N, Ramos Murguialday A, Weber C, Montoya P: Neurofeedback and brain-computer interface clinical applications. Int Rev Neurobiol 2009;86:107-117.

Cacciaglia R, Pohlack ST, Flor H, Nees F: Dissociable roles for hippocampal and amygdalar volume in human fear conditioning. Brain Struct Funct 2014;6:ahead of print.

Conrod PJ, O'Leary-Barrett M, Newton N, Topper L, Castellanos-Ryan N, Mackie C, Girard A: Effectiveness of a selective, personality-targeted prevention program for adolescent alcohol use and misuse: a cluster randomized controlled trial. JAMA Psychiatry 2013;70:334-342.

Craske MG, Treanor M, Conway CC, Zbozinek T, Vervliet B: Maximizing exposure therapy: an inhibitory learning approach. Behav Res Ther 2014;58:10-23.
Cuthbert BN, Insel TR: Toward the future of psychiatric diagnosis: the seven pillars of RDoc. BMC Med 2013; 11:126.

Cuthbert BN, Kozak MJ: Constructing constructs for psychopathology: the NIMH Research Domain Criteria. J Abnorm Psychol 2013;122:928-937.

De Raedt R, Vanderhasselt MA, Baeken C: Neurostimulation as an intervention for treatment resistant depression: from research on mechanisms towards targeted neurocognitive strategies. Clin Psychol Rev 2014;pii: S0272-7358(14)00152-4.

Diers M, Yilmaz P, Rance M, et al.:Treatment-related changes in brain activation in patients with fibromyalgia syndrome. Exp Brain Res 2012;218:619-628.

Ebner-Priemer UW, Mauchnik J, Kleindienst N, Schmahl C, Peper M, Rosenthal MZ, Flor H, Bohus M: Emotional learning during dissociative states in borderline personality disorder. J Psychiatry Neurosci 2009;34:214-222.

Everitt BJ, Robbins TW: Neural systems of reinforcement for drug addiction: from actions to habits to compulsion. Nat Neurosci 2005;8:1481-1489.
Flor H: Psychological pain interventions and neurophysiology: implications for a mechanism-based approach. Am Psychol 2014;69:188-196.

Flor H, Turk DC: An Integrated Biobehavioral Aproach to Chronic Pain and its Management. Washington, IASP Press, 2011.

Foell J, Bekrater-Bodmann R, Diers M, Flor H: Mirror therapy for phantom limb pain: brain changes and the role of body representation. Eur J Pain 2014;18:729739.

Grüsser SM, Heinz A, Raabe A, Wessa M, Podschus J, Flor $\mathrm{H}$ : Stimulus-induced craving and startle potentiation in abstinent alcoholics and controls. Eur Psychiatry 2002;17:188-193.

Grüsser SM, Wrase J, Klein S, Hermann D, Smolka MN, Ruf M, Weber-Fahr W, Flor H, Mann K, Braus DF, Heinz A: Cue-induced activation of the striatum and medial prefrontal cortex is associated with subsequent relapse in abstinent alcoholics. Psychopharmacology 2004;175:296-302. 
Hofmann SG, Asmundson GJ: Acceptance and mindfulness-based therapy: new wave or old hat? Clin Psychol Rev 2008;28:1-16.

Holmes EA, Craske MG, Graybiel AM: Psychological treatments: a call for mental-health science. Nature 2014; 511:287-289.

Lilienfeld SO: The Research Domain Criteria (RDoC): an analysis of methodological and conceptual challenges. Behav Res Ther 2014;62:129-139.

Kirsch M, Gruber I, Kirsch P, Kiefer, F: Kann AlkoholCraving durch Echtzeit fMRT-Neurofeedback beein flusst werden? Sucht 2014;60:88-89.

Krugers HJ, Joëls M: Long-lasting consequences of early life stress on brain structure, emotion and cognition. Curr Top Behav Neurosci 2014;18:81-92.

Kunkel A, Kopp B, Müller G, Villringer K, Villringer A Taub E, Flor H: Constraint-induced movement therapy for motor recovery in chronic stroke patients. Arch Phys Med Rehabil 1999;80:624-628.

Loeber S, Croissant B, Heinz A, Mann K, Flor H: Cue exposure in the treatment of alcohol dependence: effects on drinking outcome, craving and self-efficacy. $\mathrm{Br} \mathrm{J}$ Clin Psychol 2006;45:515-529.

Loeber S, Croissant B, Nakovics H, Zimmer A, Georgi A, Klein S, Diener C, Heinz A, Mann K, Flor H: The startle reflex in alcohol-dependent patients: changes after cognitive-behavioral therapy and predictive validity for drinking behavior. A pilot study. Psychother Psychosom 2007;76:385-390.

McHugh RK, Whitton SW, Peckham AD, Welge JA, Otto MW: Patient preference for psychological vs pharmacologic treatment of psychiatric disorders: a meta-analytic review. J Clin Psychiatry 2013;74:595-602.
Meaney MJ, Szyf M: Environmental programming of stress responses through DNA methylation: life at the interface between a dynamic environment and a fixed ge nome. Dialogues Clin Neurosci 2005;7:103-123.

Miller GA: Mistreating psychology in the decades of the brain. Perspect Psychol Sci 2010;5:716-743.

Miltner WH, Bauder H, Sommer M, Dettmers C, Taub E: Effects of constraint-induced movement therapy on patients with chronic motor deficits after stroke: a replication. Stroke 1999;30:586-592.

Nees F, Tzschoppe J, Patrick CJ, Vollstadt-Klein S, Steiner S, Poustka L, Banaschewski T, Barker GJ, Buchel C, Conrod PJ, Garavan H, Heinz A, Gallinat J, Lathrop M, Mann K, Artiges E, Paus T, Poline JB, Robbins TW, Rietschel M, Smolka MN, Spanagel R, Struve M, Loth E, Schumann G, Flor H, and the IMAGEN Consortium: Determinants of early alcohol use in healthy adolescents: the differential contribution of neuroimaging and psychological factors. Neuropsychopharmacology 2012;37:986-995.

Nees F, Heinrich A, Flor, H: A mechanism-oriented approach to psychopathology: the role of pavlovian conditioning. Int J Psychophysiology 2015; in press.

-Nilsen DM, Gillen G, Geller D, Hreha K, Osei E, Saleem GT: Effectiveness of interventions to improve occupational performance of people with motor impairments after stroke: an evidence-based review. Am J Occup Ther 2015;69:6901180030p1-9.

Perez-Rodriguez M, Mahon K, Russo M, Ungar AK, Burdick KE: Oxytocin and social cognition in affective and psychotic disorders. Eur Neuropsychopharmacol 2015;25:265-282.
Rabinak CA, Phan KL: Cannabinoid modulation of fear extinction brain circuits: a novel target to advance anxiety treatment. Curr Pharm Des 2014;20:2212-2217.

Rector NA, Man V, Lerman B: The expanding cognitivebehavioural therapy treatment umbrella for the anxiety disorders: disorder-specific and transdiagnostic approaches. Can J Psychiatry 2014;59:301-309.

Robinson TE, Berridge KC: The neural basis of drug craving: an incentive-sensitization theory of addiction. Brain Res Brain Res Rev 1993;18:247-291.

Rodrigues H, Figueira I, Lpes A, Gonçalves R, Mendlowicz MV, Coutinho ES, Ventura P: Does D-cycloserine enhance exposure therapy for anxiety disorders in humans? A meta-analysis. PLoS One 2014;9:e93519.

Soekadar SR, Birbaumer N, Slutzky MW, Cohen LG: Brainmachine interfaces in neurorehabilitation of stroke. Neurobiol Dis 2014;pii:S0969-9961(14)00371-4.

Thieme K, Turk DC, Flor H: Responder criteria for operant and cognitive-behavioral treatment of fibromyalgia syndrome. Arthritis Rheum 2007;57:830-836.

Wessa M, Flor H: Failure of extinction of fear responses in posttraumatic stress disorder: evidence from secondorder conditioning. Am J Psychiatry 2007;164:16841692.

Wölfling K, Mörsen CP, Duven E, Albrecht U, Grüsser SM, Flor $\mathrm{H}$ : To gamble or not to gamble: at risk for craving and relapse - learned motivated attention in pathological gambling. Biol Psychol 2011;8:275-281

\section{Imprint}

ISSN Print Edition: 1016-6262

ISSN Online Edition: 1423-0402

Journal Homepage: http://www.karger.com/ver

Publication Data: Volume 25, 2015 of 'Verhaltenstherapie' appears with 4 issues.

Copyright: ๑ 2015 by S. Karger Verlag für Medizin und Naturwissenschaften GmbH, Freiburg (Germany). All rights reserved. No part of the journal may be repro-duced in any form without the written permission of the publisher. This includes digitalisation and any further electronic computing, like saving, copying, printing or electronic transmission of digitalized material from this journal (online or offline). Authorization to photocopy items for internal or personal use of specific clients is granted by Karger.

Photocopying: This journal has been registered with the Copyright Clearance Center (CCC), as indicated by the code appearing on the first page of each article. For readers in the US, this code signals consent for copying of articles for personal or internal use, or for the personal or internal use of specific clients, provided that the stated fee is paid per copy directly to Copyright Clearance Center Inc., 222 Rosewood Drive, Danvers, MA 01923 (USA).

A copy of the first page of the article must accompany payment. Consent does not extend to copying for general distribution, for promotion, for creating new works, or for resale. In these cases, specific written permission must be obtained from the copyright owner, S. Karger GmbH, Wilhelmstraße 20A, 79098 Freiburg (Germany).

Disclaimer: The statements and data contained in this publication are solely those of the individual authors and contributors and not of the publisher and the editor(s). The appearance of advertisements in the journal is not a warranty, endorsement, or approval of the products or services advertised or of their effectiveness, quality or safety. The publisher and the editor(s) disclaim responsibility for any injury to persons or property resulting form any ideas, methods, instructions or products referred to in the content or advertisements.

Distribution and Subscription: Karger offers three types of subscription: Print Only, Online Only and the combined Print + Online. The basic annual subscription rate is the same for all three delivery forms; however, a fee for the combined print and online subscription is levied, and there is a postage and handling charge for Print Only and Print + Online. Subscriptions run for a full calendar year. Prices are given per volume.
Print subscription: EUR 152.- + postage and handling.

Online subscription: EUR 152.-.

Combined (print + online) subscription: EUR 202.- + postage and handling.

For customers in Germany: Please turn to your bookshop or to

S. Karger Verlag für Medizin und Naturwissenschaften $\mathrm{GmbH}$

Wilhelmstr. 20A, 79098 Freiburg (Germany)

Tel. +497614520 70, Fax +497614520714

E-mail information@karger.com

For customers in all other countries: Please contact your bookshop or S. Karger AG, Allschwilerstr. 10, 4009 Basel (Switzerland)

Tel. +416130611 11, Fax +41613061234

E-mail karger@karger.com

Advertising: Correspondence should be addressed to the publisher.

S. Karger Verlag für Medizin und Naturwissenschaften GmbH

Attn. Ellen Zimmermann (Head of Marketing)

E-mail e.zimmermann@karger.com

Price list No. 20 of January 1, 2015 is effective.

V.i.S.d.P. (Person responsible according to the German Press Law): Sibylle Gross

Type setting and printing: Kraft Druck GmbH, 76275 Ettlingen, Germany.

Bibliographic Services

Current Contents/Social \& Behavioral Sciences

Science Citation Index (SCI)

Social Science Citation Index (SSCI)

PsycINFO

ISBN: 978-3-318-05450-7

eISBN: 978-3-318-05451-4

\section{KARGER}

Fax +4976145207 14 Information@Karger.com www.karger.com

\section{2015 S. Karger GmbH, Freiburg}

Accessible online at:

www.karger.com/ver 